

\title{
SERVICIOS EDUCATIVOS MEDIANTE LA UTILIZACIÓN DE TECNOLOGÍAS de Cloud Computing
}

\author{
Manuel Yrigoyen Quintanilla \\ Carlos Torres Paredes
}

\section{Resumen}

Cloud Computing es un paradigma que define un nuevo modelo de prestación, uso y entrega de los servicios de tecnologías de la información (TI). Al utilizarlo las organizaciones podrán satisfacer sus necesidades de información sin tener que comprar o añadir equipos, software ni personal. A través de la 'nube', los usuarios de los recursos de TI podrán acceder, bajo demanda, a un gran número de recursos informáticos virtualizados asignados dinámicamente, dotándose así de una enorme capacidad de procesamiento y almacenamiento sin que se requiera instalar una infraestructura de forma local, lo que se traduce en considerables beneficios. Asimismo, Cloud Computing representa una oportunidad para repensar el modelo de operación de las Tlen el ámbito universitario, que permita enfocarse en la prestación de los servicios educativos más que en la infraestructura tecnológica que la soporta. Por la complejidad de las tecnologías inherentes al modelo, es necesaria la utilización de un marco de trabajo (framework) que guíe el análisis. En este contexto, se examina, propone y describe un marco de trabajo específico para definir una Nube Académica Universitaria (NAU).

Palabras clave: Cloud Computing / software como servicio / plataforma como servicio / infraestructura como servicio / servicios educativos / gobierno de las tecnologías de información, marcos de trabajo, COBIT, ITIL. 


\section{Introducción}

El presente artículo está basado en el estudio de investigación patrocinado por el Instituto de Investigación Científica (IDIC) de la Universidad de Lima, desarrollado en el 2010, y que buscó determinar si las tecnologías de Cloud Computing están lo suficientemente maduras para que puedan ser utilizadas en el ámbito académico universitario, recomendando, de ser el caso, una estrategia de implantación y los primeros pasos o tareas a realizar para lograr el objetivo.

En ese contexto, se inició el estudio haciendo una revisión bibliográfica respecto del estado del arte de Cloud Computing, identificando los modelos de servicio que soporta, su arquitectura, estándares y otros componentes relevantes. También se efectuaron pruebas de concepto para determinar la viabilidad de utilizar los servicios educativos identificados en el ámbito universitario, en la etapa inicial del proyecto.

Se concluyó que, no obstante las tecnologías relacionadas con Cloud Computing se encuentran en plena evolución, están lo suficientemente maduras para que las instituciones universitarias las comiencen a utilizar de modo gradual, por lo que se recomienda una estrategia en dos etapas: una piloto y otra de iniciación.

A continuación se plantearon las estrategias a seguir y los servicios a implantar en la etapa piloto, utilizando los modelos de nube pública y nube privada. Esta etapa tendría una duración de un año.

Para la etapa de iniciación, posterior a la etapa piloto, se plantea efectuar una mejora de los procesos de TI relevantes, relacionados con la implantación de servicios educativos bajo el modelo de nube pública, con el fin de que las instituciones educativas estén mejor preparadas para afrontar este proceso. Esta tarea se efectuó proponiendo un modelo de procesos con ayuda del marco de trabajo COBIT 4.1, utilizando las herramientas de los Modelos de Madurez y Prácticas de Control en la determinación de los proyectos de mejora de los procesos de TI seleccionados, que denominamos el mapa de ruta para la implantación de la Nube Académica pública.

\section{Estado del arte de las tecnologías de Cloud Computing}

\subsection{Definición de Cloud Computing}

Cloud Computing es un nuevo campo de investigación multidisciplinario, que se considera será la evolución y convergencia de algunas tendencias independientes como la computación utilitaria, la elasticidad, la virtualización, la computación en 
grilla (grid computing), la computación distribuida, la externalización (outsourcing) de contenidos, el almacenamiento, la seguridad, la Web 2.0 y otras (Pallis 2010: 70).

Aun cuando el paradigma del Cloud Computing está en plena evolución, hay organismos internacionales que están estandarizando conceptos y definiciones. En este sentido, el National Institute of Standards and Technology (NIST) publica en su sitio web los conceptos que resumiremos a continuación, como resultado del "Seminario de Cloud Computing" que llevó a cabo en mayo del 2010:

Cloud Computing es un modelo para habilitar el acceso, conveniente y bajo demanda, a la red de un pool compartido de recursos de cómputo configurables (redes, servidores, almacenamiento, aplicaciones y servicios) que pueden ser rápidamente proporcionados y retirados con un mínimo esfuerzo de administración o interacción con el proveedor del servicio (Leaf 2010: 4) (traducción nuestra)

Existen diversas definiciones de lo que se conoce como 'nube', entre las que se encuentra la siguiente:

Una 'nube' es un tipo de data center distribuida, la cual proporciona infraestructura de TI como servicios. Dispone de recursos en forma masiva, los cuales son proporcionados a los usuarios de manera sencilla, dinámica, flexible y elástica, permitiendo además un monitoreo en tiempo real de los servicios recibidos (IEEE 2009: 125) (traducción nuestra).

Cloud Computing tiene en general cinco características: autoservicio bajo demanda, acceso de red ubicuo, medición del uso, elasticidad y pool de recursos.

Usualmente, se efectúa en escala masiva con homogeneidad de equipos y software. Descansa sobre todo en las tecnologías de virtualización, computación de alta disponibilidad y software libre de bajo costo. Se encuentra muy disperso geográficamente, y enfocado más en los servicios que en los sistemas. Como involucra la compartición de recursos, esta demanda tecnologías avanzadas de seguridad (Badger y Grance 2010: 4). 


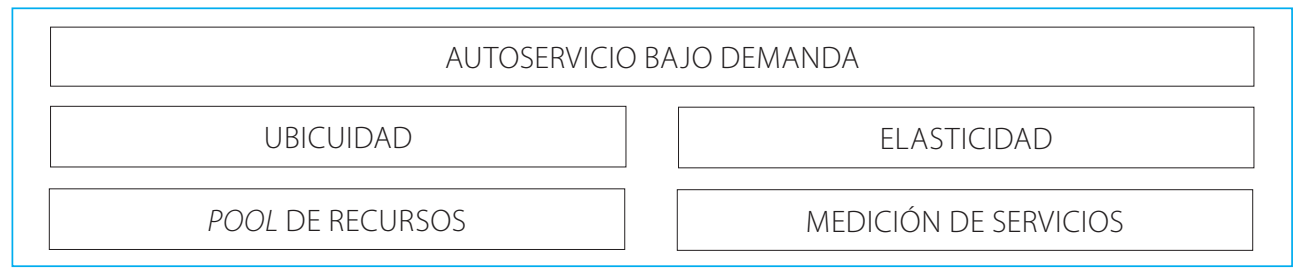

CARACTERÍSTICAS COMUNES

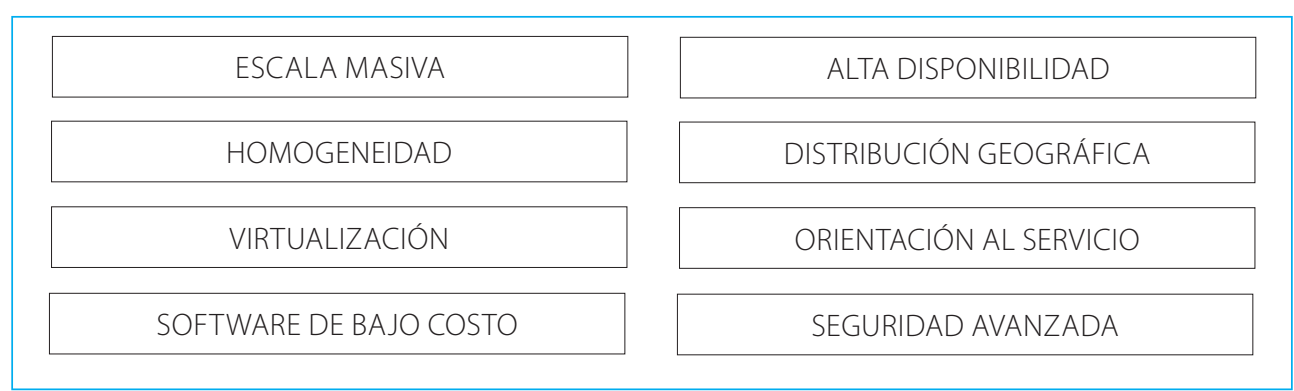

Figura 1. Características de la nube

Fuente: Tseronis et al. (2010: 15).

\subsection{Modelos de servicio}

Según Badger y Grance (2010: 6), Cloud Computing tiene tres principales modelos de servicio, que son:

- Software como servicios (Software as a Service - SaaS).- En este modelo el proveedor de servicios proporciona la aplicación que necesita el usuario, pero teniendo el control total de los recursos. El usuario posee un control limitado en la administración de las aplicaciones. De igual manera existen limitaciones para mover datos de la aplicación, de un proveedor a otro, así como limitaciones para la compartición de datos, situación sencilla en un computador con software propio.

- Plataforma como servicios (Platform as a Service - PaaS)

Proporciona las herramientas para el desarrollo de aplicaciones por parte de los usuarios, y la infraestructura donde se podrán ejecutar dichas aplicaciones. En este modelo el proveedor tiene el control total sobre la infraestructura (hardware, sistema operativo) y el control administrativo sobre las aplicaciones desarrolladas. 
Servicios educativos mediante la utilización de tecnologías de Cloud Computing

Se restringe la habilidad para guardar datos en un formato portable y las herramientas de desarrollo son de propiedad del proveedor de servicios, no compatibles con otras herramientas de mercado.

- Infraestructura como servicios (Infrastructure as a Service-laaS)

El proveedor proporciona servicios de computación y almacenamiento, en el cual el cliente tiene el control total sobre la aplicación, las herramientas de desarrollo (middleware) y el sistema operativo. Este modelo tiene aspectos de seguridad que deben ser contemplados cuando los datos ingresan a la nube del proveedor.

\subsection{Arquitectura}

Viene a ser la arquitectura de nube (Cloud Architecture). Consiste de tres capas o niveles abstractos, que son: infraestructura, plataforma y aplicación (Pallis 2010: 71).

- Infraestructura.- Es el nivel más bajo, y el encargado de proporcionar el procesamiento, el almacenamiento, las redes y otros recursos fundamentales de cómputo, como los servicios estandarizados sobre la red. Los clientes pueden desplegar y correr sistemas operativos y software sobre esta infraestructura.

- Plataforma.- Proporciona mayor abstracción y servicios para desarrollar probar, desplegar y almacenar aplicaciones en el mismo ambiente de desarrollo integrado. Esta capa proporciona el ambiente donde correrán las aplicaciones y el medio para desplegarlas usando lenguajes de programación y herramientas que soporta el proveedor de la nube.

- Aplicación.- Es el nivel más alto y proporciona una aplicación completa ofrecida como servicio.

Una capa de interfaz de usuario será necesaria para poder interactuar de la manera más adecuada con los recursos de la nube.

A continuación se muestra un esquema de la arquitectura por capas del Cloud Computing, en la que se pueden observar los elementos descritos:

- Infraestructura

- Plataforma

- Aplicaciones

- La interfaz de usuario 


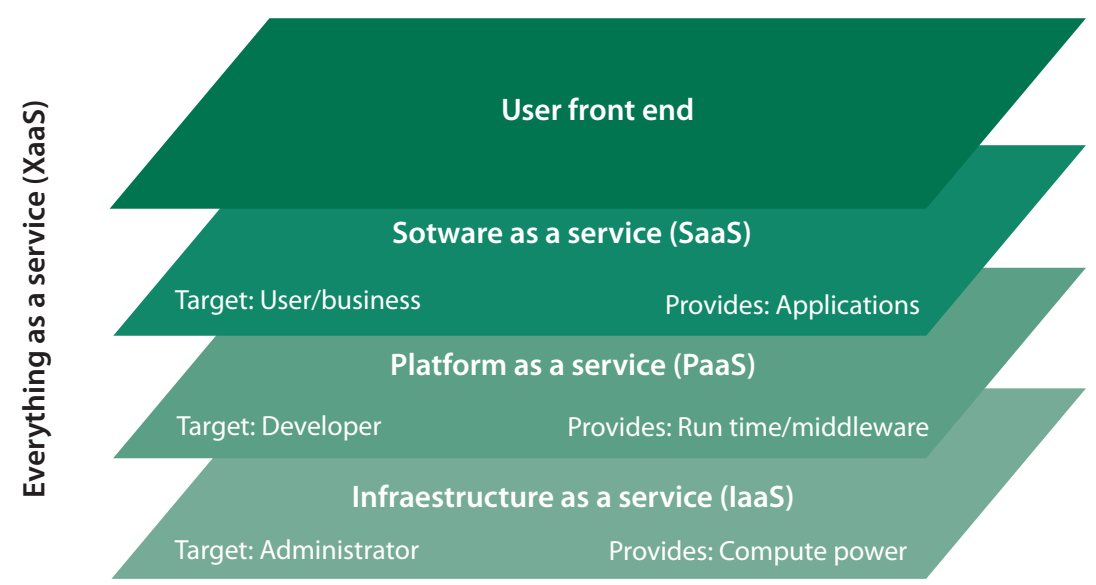

Figura 2. Arquitectura por capas de la nube

Fuente: Pallis (2010: 71).

\subsection{Modelos de despliegue}

Actualmente se vienen experimentando cuatro esquemas de implantación (Badger y Grance 2010: 7), que son los siguientes:

- Nube privada.- En este esquema los recursos de las TI se encuentran dentro de la organización, pero se optimiza su gestión y operación mediante la aplicación de las tecnologías de Cloud Computing. Este esquema es utilizado cuando la organización dispone de una infraestructura importante de las TI o cuando los servicios que se requieren no están disponibles en la nube pública.

- Nube cooperativa.- Es un conjunto de nubes privadas con una administración común. Se aplica a sociedades de intereses comunes.

- Nube pública.-Cuando los recursos de TI se encuentran fuera del ámbito de las organizaciones y son proporcionadas por proveedores de servicios de Cloud Computing.

- Nube híbrida.-Es la combinación de los esquemas público y privado definida en los párrafos anteriores. 
Servicios educativos mediante la utilización de tecnologías de Cloud Computing

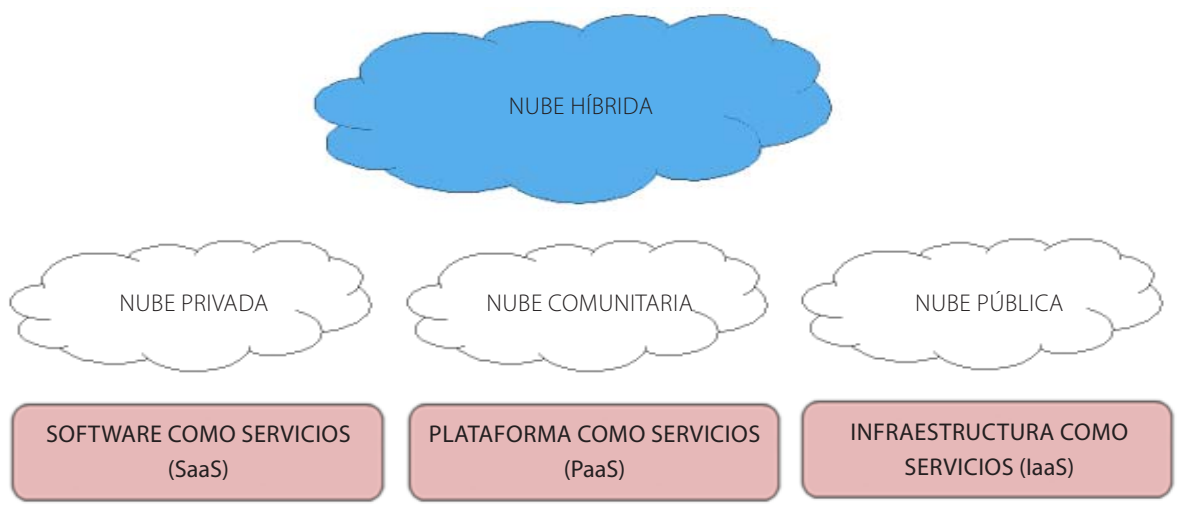

Figura 3. Modelos de servicio y despliegue

Fuente: Tseronis et al. (2010: 15).

\subsection{Proveedores y servicios}

En base a nuestra investigación hemos podido determinar los servicios y proveedores más representativos en el esquema de nube pública, los que se muestran a continuación, clasificados según su modelo de servicio.

- Software como servicios (SaaS)

- Google Apps, servicios de colaboración y comunicación para el ámbito empresarial (http://www.google.com/a).

- Microsoft Live@edu, servicios de colaboración y comunicación para el ámbito educativo (http://www.microsoft.com/liveatedu).

- IBMCloud Academy, servicios de colaboración y comunicación para el ámbito educativo (http://www.ibm.com/solutions/education/cloudacademy).

- Salesforce, CRM en demanda (http://www.salesforce.com).

- Virtual SAP, ERP en demanda (http://virtualsap.com).

- Oracle CRM On Demand, CRM en demanda (http://crmondemand.oracle. com).

- Basecamp, gestión de proyectos a través de la web (http://basecamphq. com).

- Quickbase, base de datos personalizable disponible en línea (http:// quickbase.intuit.com).

- Plataforma como servicios (PaaS)

- Windows Azure, de Microsoft 
- Google App Engine, de Google

- BlueCloud, Websphere, CloudBurst Appliance, Research Compute Cloud (RC2), de IBM

- Force.com, de Salesforce.com

- Infraestructura como servicios (laaS)

- Elastic Computing Cloud (EC2), Simple Storage Service (S3), Simple Queue Service, SimpleDB, de la compañía Amazon.

\section{Estrategia de implantación de los servicios educativos utilizando tecnologías de Cloud Computing}

Las TI son un componente crítico para el funcionamiento de las instituciones académicas universitarias. Independientemente de cuán bien administrados estén sus recursos de $\mathrm{Tl}$, sus costos totales tienden a crecer debido al incremento del empleo de nuevas aplicaciones intensivas, en el uso de la voz, el video, los modelos de simulación, el análisis de datos y otras por parte de la comunidad universitaria, las cuales requieren de gran capacidad de procesamiento y de ancho de banda. De la misma forma, los costos de administración de estos recursos tienden a crecer, y en mayor medida en aquellas instituciones que siguen la tradición de construir y operar sus propios sistemas de información. La pregunta es si las universidades van a poder continuar con este modelo de operación de un ambiente único de las $\mathrm{Tl}$ enorme y caro que viene funcionando hace mucho tiempo. En este contexto, muchas instituciones están buscando nuevos modelos que les permita ser más eficientes y competitivas, y enfocarse en su misión y en sus competencias clave. Cloud Computing representa una oportunidad para repensar el modelo de operación de las $\mathrm{TI}$ en el ámbito universitario, posibilitando enfocarse en la prestación de los servicios educativos más que en la infraestructura tecnológica que la soporta (Educase/Nacubo 2010: 2).

Uno de los elementos más importantes a considerar a la hora de utilizar las tecnologías de Cloud Computing es la reducción del costo por la utilización del hardware y software. Porque pagar por usar la suite Office de MicroSoft cuando se puede usar una suite libre de costo basada en web como las ofrecidas por Google (Google Docs) o por la misma Microsoft (Live@edu). Adicionalmente, no solo las aplicaciones son ofrecidas libremente, sino también la capacidad de almacenamiento. Con Cloud Computing, en vez de guardar contenidos en grandes espacios de disco dentro de las entidades educativas, las facultades y los estudiantes pueden guardarlos en los servicios de la nube. Debido a que las aplicaciones y los datos son almacenados en la web, ellos pueden ser accedidos con dispositivos pequeños y baratos como nunca antes. Una netbook o una laptop de baja potencia, con una capacidad limitada de procesamiento y almacenamiento, es tan útil 
como una computadora de escritorio más rápida. Aun los teléfonos inteligentes pueden ser lo suficientemente potentes para acceder a muchos de los servicios de Cloud. Estas aplicaciones web eliminan la necesidad de que las facultades y los estudiantes tengan un software específico en sus computadoras. El único software que se necesita es un browser libre como el Firefox, el Safari o el Internet Explorer (Holschuh y Caverly 2010: 36).

En base a las investigaciones efectuadas consideramos que las instituciones universitarias deben tomar la decisión de utilizar las nuevas tecnologías de Cloud Computing, en todos sus ámbitos de actuación, priorizando los referidos a las actividades educativas.

Por estar esta tecnología en plena evolución, consideramos conveniente comenzar el proceso de adopción con una etapa piloto o de experimentación, seguida de una etapa de iniciación, en la que en base a la experiencia y los conocimientos ganados en la etapa piloto se implantarían los servicios más adecuados para los diferentes requerimientos del claustro.

Para la etapa piloto haremos una selección de los servicios a implantar en calidad de prueba, mientras que para la etapa de iniciación definiremos los proyectos o actividades necesarias para llevar este proceso de la mejor manera posible, usando para ello el marco de referencia de COBIT.

\subsection{Etapa piloto}

A continuación exponemos los servicios de Cloud Computing a implementar en esta etapa, cuya duración no debe ser mayor a un año.

\subsubsection{Nube pública}

Consideramos que en este esquema de implantación se debe experimentar con los tres modelos existentes, de acuerdo a los siguientes lineamientos:

\section{A) Software como servicios}

Lo más importante a considerar respecto de los servicios educativos es si las aplicaciones serán ejecutadas en casa o en la nube. Cuando los procesos son efectuados en casa, se tiene todo el control sobre estos, pero con limitadas oportunidades para aplicar economías de escala. Cuando el proceso se encuentra en la nube, decrece el control, pero se incrementan las oportunidades de lograr economías de escala (Katzan 2010: 39).

Los servicios de Cloud Computing comerciales se están masificando y convirtiéndose en un commodity. Diversas modalidades de servicio, como 
software como servicios (SaaS), infraestructura como servicios (laaS), plataforma como servicios (PaaS), entre otras, están creciendo en capacidad y complejidad. Sin embargo, todavía hay pocos servicios que den un soporte directo a las actividades académicas universitarias (Hurwitz et al. 2010: 18-22).

En nuestro proceso de investigación aún no hemos podido encontrar aplicaciones orientadas al ámbito académico y de apoyo al proceso enseñanzaaprendizaje. Las principales aplicaciones disponibles en este rubro son las aplicaciones ofimáticas (procesamiento de texto, hojas de cálculo, etcétera), de trabajo en equipo y de correo electrónico.

Por este motivo, consideramos que las aplicaciones o servicios a implantar en la etapa piloto, y que estarán a disposición de toda la comunidad académica (alumnos, profesores e investigadores), constituyen los dos servicios más representativos de este tipo de aplicaciones, que a la fecha son:

- Servicios de colaboración y comunicación de Google Apps, de Google.

- Servicios de colaboración y comunicación de Live@edu, de MicroSoft.

B) Plataforma como servicios

Debido a que las capacidades que se tieneen este modelo están relacionadas con el desarrollo y despliegue de aplicaciones, consideramos que las universidades que cuenten con facultades de Ingeniería de Sistemas, Computación, Informática, o áreas afines, deben experimentar con estos servicios, los cuales deberían estar disponibles para los alumnos, profesores e investigadores. En base a la investigación efectuada, consideramos que los servicios a implantar en la etapa de experimentación sean los dos más representativos del mercado:

- Google App, de la compañía Google, y

- MicroSoft Azure, de la compañía MicroSoft

C) Infraestructura como servicios

Este servicio puede ser utilizado por toda la comunidad académica (alumnos, profesores e investigadores), en su rol de proveedor de recursos de almacenamiento, con el fin de que los usuarios puedan extender su capacidad de almacenamiento de contenidos.

Asimismo, pueden ser utilizados por las facultades de Ingeniería de Sistemas o áreas afines — por alumnos, profesores e investigadores-, en su rol de proveedor de recursos de cómputo (procesamiento, almacenamiento, redes, etcétera) a fin de utilizarla para experimentar con la infraestructura de la nube, teniendo el control sobre los sistemas operativos, el almacenamiento y el despliegue de aplicaciones, así como un control limitado sobre los componentes de la red. En base a nuestra investigación, hemos determinado que los servicios a probar en la etapa piloto sean los más representativos, los que se indican a continuación: 
- Elastic Computing Cloud (EC2), de Amazon, y

- Simple Storage Service (S3), de Amazon.

A continuación se muestra un esquema general de la etapa piloto con nube pública:

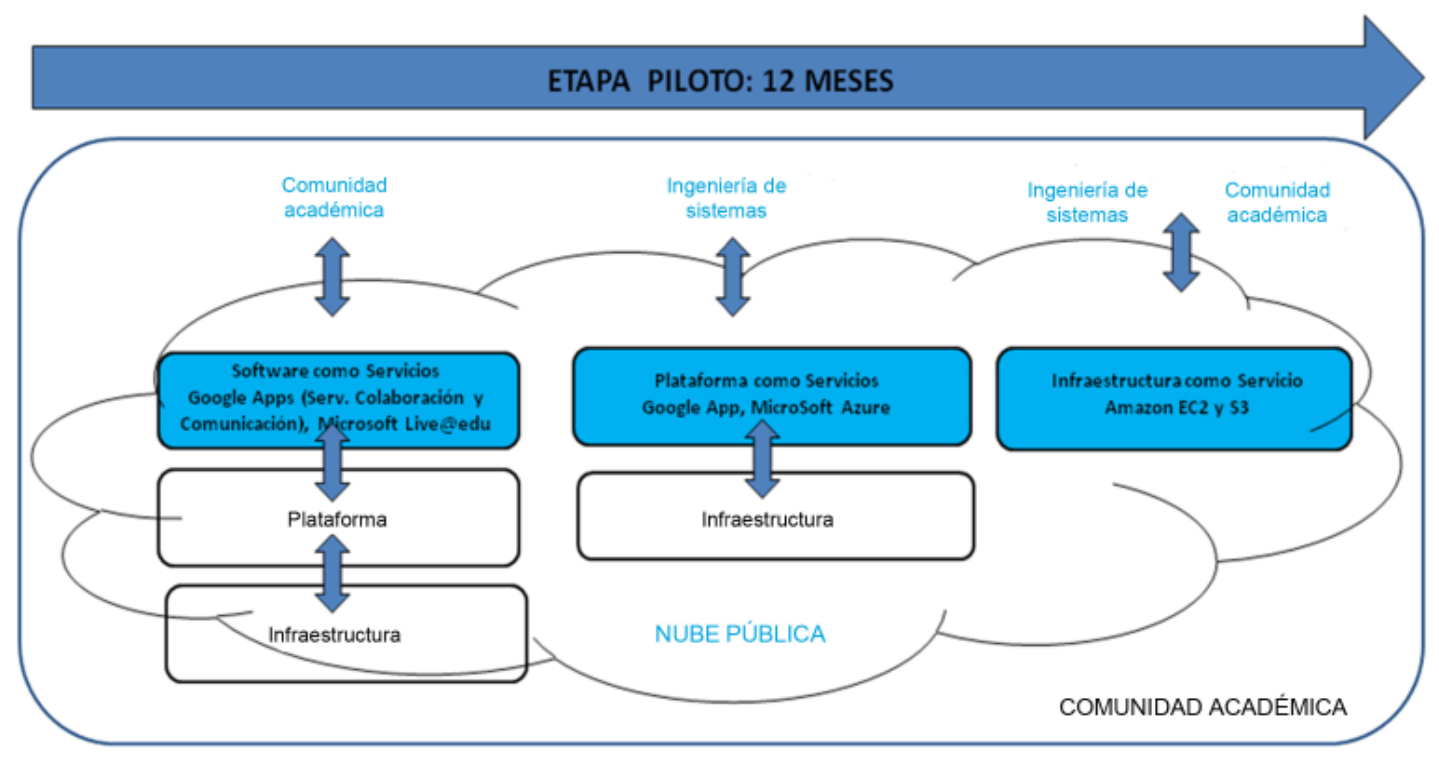

Figura 4. Etapa piloto con nube pública

Elaboración propia.

\subsubsection{Nube privada}

Al igual que en el caso de la nube pública, consideramos que las instituciones académicas universitarias deben comenzar con una etapa de experimentación de las tecnologías de Cloud Computing, aplicadas en su infraestructura de TI, creando una nube privada que se irá desarrollando y fortaleciendo con el tiempo.

Esta nube será utilizada para centralizar y ofrecer como servicios distintas aplicaciones necesarias para fortalecer el proceso enseñanza-aprendizaje, que estén fuera del alcance de las aplicaciones ofimáticas, de trabajo en equipo y de correo electrónico cubiertos por la nube pública.

De esta forma, el alumno tendrá acceso a estos aplicativos desde cualquier lugar del campus, e incluso desde fuera de este, debiendo contar para ello con un equipo de cómputo ligero, que puede ser la computadora de la universidad, la 
propia laptop o netbook del alumno o, en algunos casos, dispositivos más pequeños como un ipad o un teléfono inteligente.

Estos dispositivos deberán contar solamente con un browser y con los permisos correspondientes para acceder a los servicios educativos de la nube.

Contando con la mencionada infraestructura será mucho más fácil y más económico para las universidades el proceso de ampliación de estos servicios, mediante la incorporación de nuevas aplicaciones y herramientas disponibles tanto en su versión libre como comercial en beneficio de la comunidad universitaria.

Otro beneficio de este nuevo enfoque de trabajo será el ahorro en la renovación de las computadoras personales de las universidades, al no requerir estas de grandes prestaciones ni capacidades, ya que la capacidad de proceso y almacenamiento estará en la nube.

\section{ETAPA PILOTO: 12 MESES}

\section{CAMPUS UNIVERSITARIO}

Aplicaciones para fortalecer el proceso enseñanzaaprendizaje

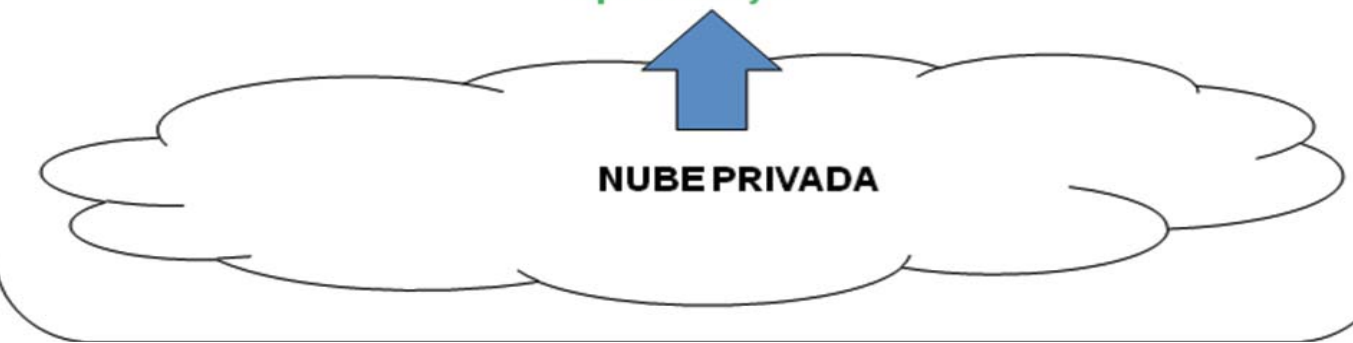

Figura 5. Etapa piloto con nube privada

Elaboración propia.

\subsection{Etapa de iniciación}

En esta etapa se desarrollan las tareas relacionadas con la selección e implantación de los servicios de Cloud Computing, en base a los resultados obtenidos durante la etapa piloto y a los estudios e investigaciones posteriores. 
Para efectos de la investigación nos enfocaremos solamente en el esquema de la nube pública, pues consideramos que este enfoque podría ser el de más popularidad en la mayoría de las universidades, no descartándose el esquema de la nube privada, que posiblemente sea implantado en forma simultánea o en una etapa posterior.

Entre los temas clave que tendrán que enfrentar las instituciones académicas universitarias en este proceso están los siguientes:

- Identificación de los servicios educativos requeridos por las universidades

- Identificación de los servicios de nube que satisfagan estos requerimientos

- Identificación y selección de los proveedores de estos servicios

- Definición de contratos para la prestación de los servicios

- Definición de los niveles de servicios requeridos

- Identificación de los recursos de TI necesarios para el uso de los servicios de la nube

- Administración de los riesgos propios de este nuevo modelo de trabajo

- Aspectos de continuidad y seguridad de los servicios

- Capacitación y entrenamiento

- Implantación gradual de los servicios seleccionados

- Monitoreo y supervisión del desempeño de los servicios

A continuación se define el concepto de Nube Académica y se selecciona un marco de trabajo (framework) que permitirá determinar un mapa de ruta a seguir en el proceso de implantación de las tecnologías de Cloud Computing en el ámbito académico universitario.

\subsubsection{Nube Académica}

Empleamos este término para referirnos a los recursos de $\mathrm{Tl}$ que utilizan tecnologías de Cloud Computing en cualquiera de sus esquemas de trabajo, formas o modalidades y que son aplicados en el ámbito universitario para dar soporte a sus actividades académicas mediante la prestación de servicios educativos. 


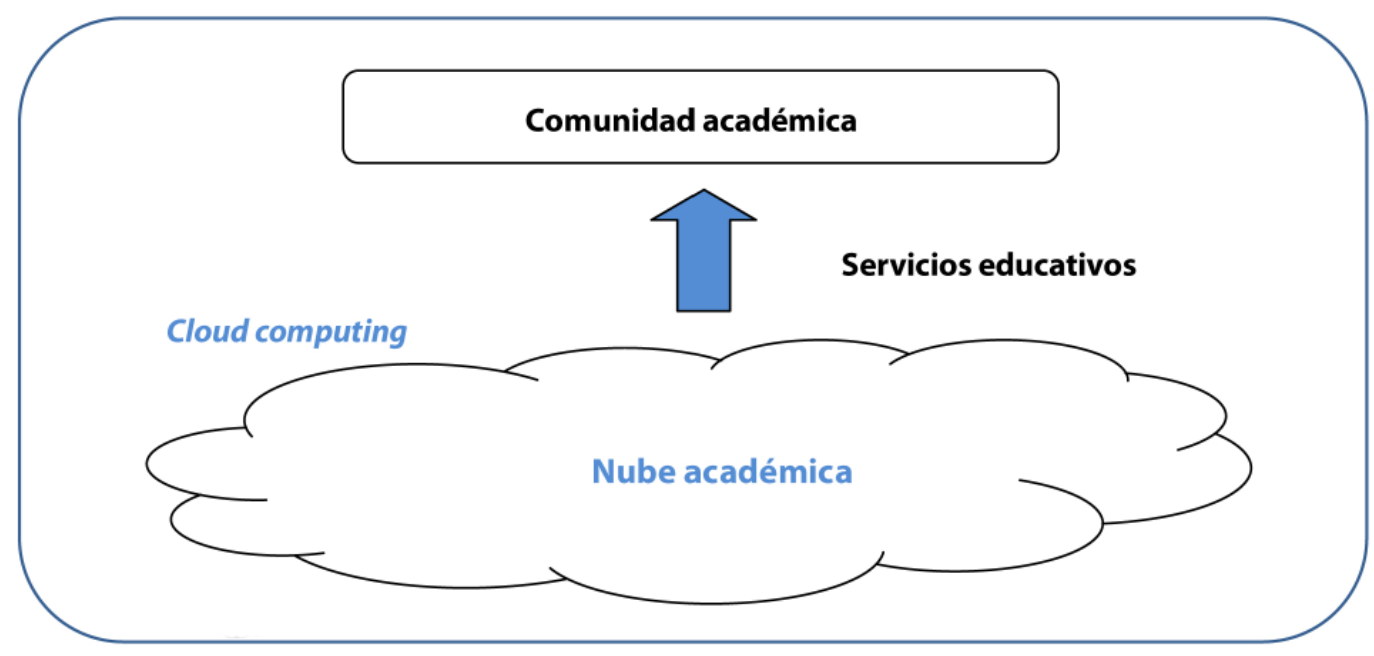

Figura 6. Nube académica

Elaboración propia.

Los servicios educativos proporcionados por la Nube Académica están dirigidos a la comunidad académica integrada por estudiantes, docentes e investigadores, con el propósito de fortalecer el proceso enseñanza-aprendizaje. Estos servicios son, entre otros, los siguientes:

- Acceso a portales universitarios

- Acceso a contenidos temáticos especializados

- Servicio de correo electrónico

- Acceso a foros especializados

- Trabajo colaborativo virtual

- Acceso a laboratorios virtuales

- Acceso a bibliotecas virtuales

- Acceso a videotecas de clases

- Acceso a blogs de profesores o alumnos

- Acceso a bases de datos especializadas

- Tutorías virtuales

- Acceso a clases virtuales (a distancia)

- Acceso a sistemas y herramientas especializadas 


\subsubsection{Marcos de trabajo (framework)}

Para definir el concepto de framework podemos referirnos al marco de trabajo de Zachman (Zachman framework), que es ampliamente utilizado para definir o documentar una arquitectura empresarial. John Zachman se basó en prácticas de la arquitectura tradicional y de la ingeniería para desarrollar su marco de trabajo, el que está compuesto de una estructura lógica para clasificar y organizar aquellos elementos de la empresa que son significativos tanto para la gerencia de la empresa como para el desarrollo de sus sistemas de información (Minoli 2008: 57).

Según Gartner:

[...] Los frameworks son importantes porque proporcionan el contexto dentro del cual el pensamiento organizacional puede ser estructurado. Un buen framework define los componentes de una arquitectura organizacional y la relación entre ellos, proporcionando al equipo de arquitectura y a la organización, de un conjunto de conceptos y semántica compartida para describir su arquitectura..." [...] (2010).

El nuevo paradigma del Cloud Computing trae inherente una gran complejidad tecnológica, sustentada en el hecho de que la infraestructura de las TI de las organizaciones podrá estar total o parcialmente en la nube, brindando los servicios que se requieran de una forma flexible, elástica, económica y sobre todo segura.

Se tendrá que definir los niveles de servicio y de disponibilidad, los esquemas de monitoreo, el desarrollo o implantación de las interfaces necesarias con los aplicativos de las organizaciones, entre otros, por lo que consideramos apropiada la utilización de un marco de trabajo (framework) o marco de referencia apropiado que nos ayude a conceptualizarlo y analizarlo.

La velocidad de desarrollo del dominio de la administración de las TI ha estimulado el desarrollo, la importancia (de otras disciplinas) y la aceptación de frameworks de gestión, métodos y metodologías que dan soporte a la administración moderna de las TI (itSMF 2006: 1, 19, 35, 149).

A continuación se analizan de manera resumida los principales marcos de trabajo que podrán ayudarnos en la tarea que nos ocupa.

- COBIT (Control Objetives for Information and Related Technology).- Es un marco de trabajo orientado al control de los procesos de las TI de las organizaciones, muy utilizado para la implantación del concepto de gobierno de las TI. Es soportado por las organizaciones ISACA (Information Systems Audit and Control Association) e ITGI (Information Technology Governance Institute). Es 
un marco de nivel estratégico de amplio alcance que ayuda a determinar los aspectos que se deben considerar ("el qué") y no a determinar cómo efectuarlos ("el cómo"), tarea posterior que puede ser complementada utilizando ITIL y los otros marcos de la ISO.

- ITIL (The IT Infraestructure Library).- Brinda una guía de buenas prácticas para la gestión de los servicios de las TI y proporciona un marco de trabajo de aceptación universal que contempla un conjunto de procesos integrados para la provisión de servicios de TI de alta calidad. Es un marco de nivel operacional, enfocado en el detalle de cómo efectuar las tareas ("el cómo").

- ISO 9000 (International Organization for Standardization).- Es un nombre genérico dado a una serie de estándares que han sido desarrollados para abordar los sistemas de administración de la calidad (QMS) dentro de una organización, con el fin de demostrar sus capacidades para satisfacer los requerimientos de sus clientes.

- 150 27000. Proporciona un modelo y una guía detallada para reducir la exposición al riesgo de la seguridad de la información de una organización.

- COSO (Committee of Sponsoring Organizations of the Treadway Commission).Está orientado a dar soporte al concepto de gobierno corporativo, y está enfocado en los aspectos de control interno de las organizaciones.

Consideramos, por lo tanto, que el marco de trabajo más adecuado para estudiar los aspectos relacionados con la implantación de la Nube Académica es el COBIT, ya que se trata de un framework de amplia aceptación que se concentra en la gestión y el control de las TI de una organización, y brinda un conjunto de buenas prácticas y una guía para fortalecer las capacidades de las TI, garantizando un alineamiento con las metas de la organización, administrando el riesgo y generando valor.

\subsubsection{Marco de trabajo para la nube pública}

Teniendo en cuenta las características y propiedades del modelo de nube pública del Cloud Computing, las características del claustro universitario, sus necesidades de información, así como los componentes y estructura del COBIT, se ha procedido a adaptarlo para definir un marco de trabajo ad hoc para el análisis de la Nube Académica. 
Este marco está compuesto por los recursos de la Nube Académica, los procesos de las TI y los servicios educativos, tal como se ilustra en el gráfico siguiente y se explica más adelante.

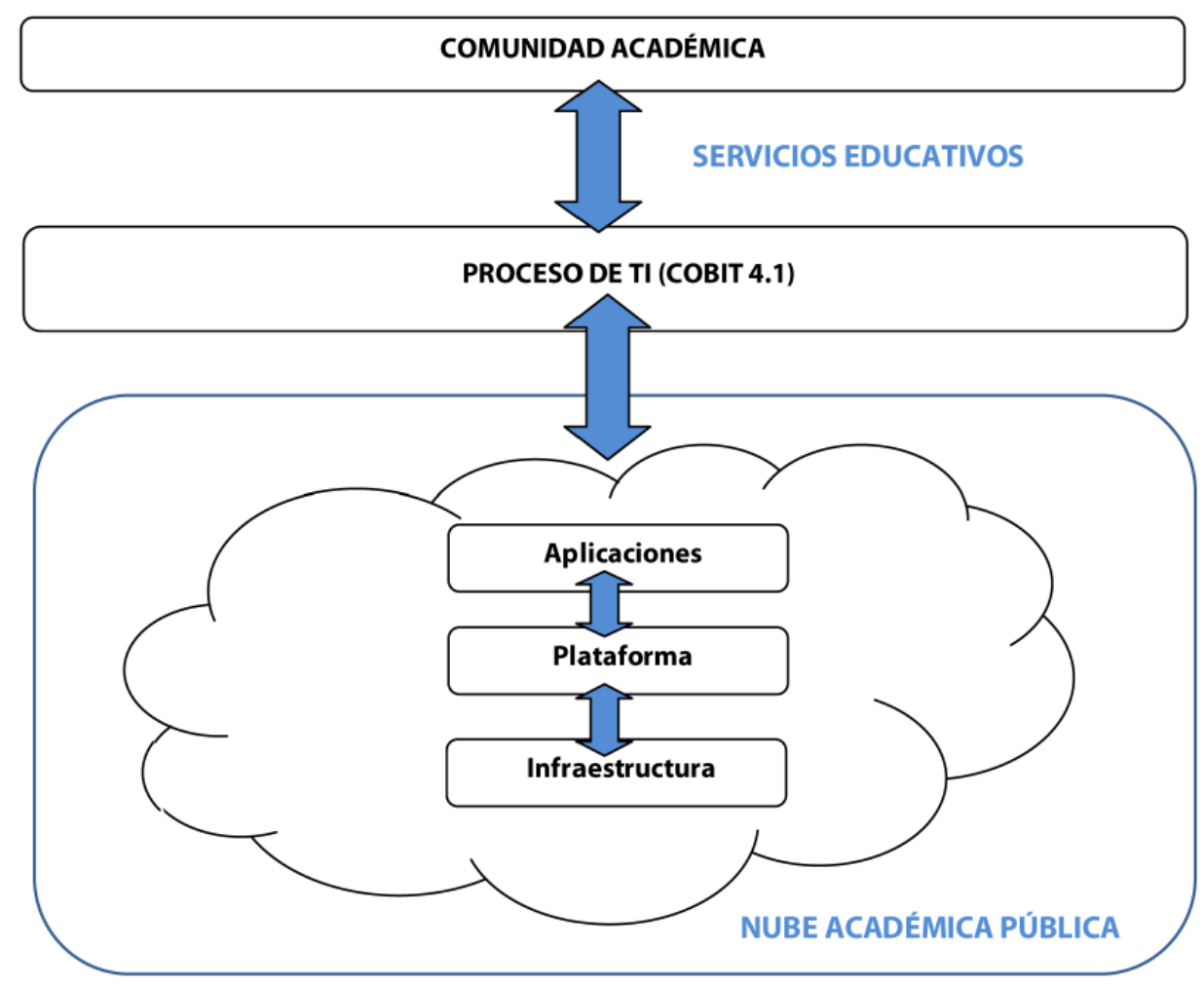

Figura 7. Arquitectura de las TI para la nube pública

Elaboración propia. 


\section{A) Recursos de la Nube Académica}

La Nube Académica está compuesta de infraestructura, plataforma y aplicaciones, conforme se definió en el acápite 1.3, referido a la arquitectura del Cloud Computing, de acuerdo con el esquema que se presenta a continuación. Los recursos de la nube, por ser de carácter público, son de entera responsabilidad del proveedor de los servicios.

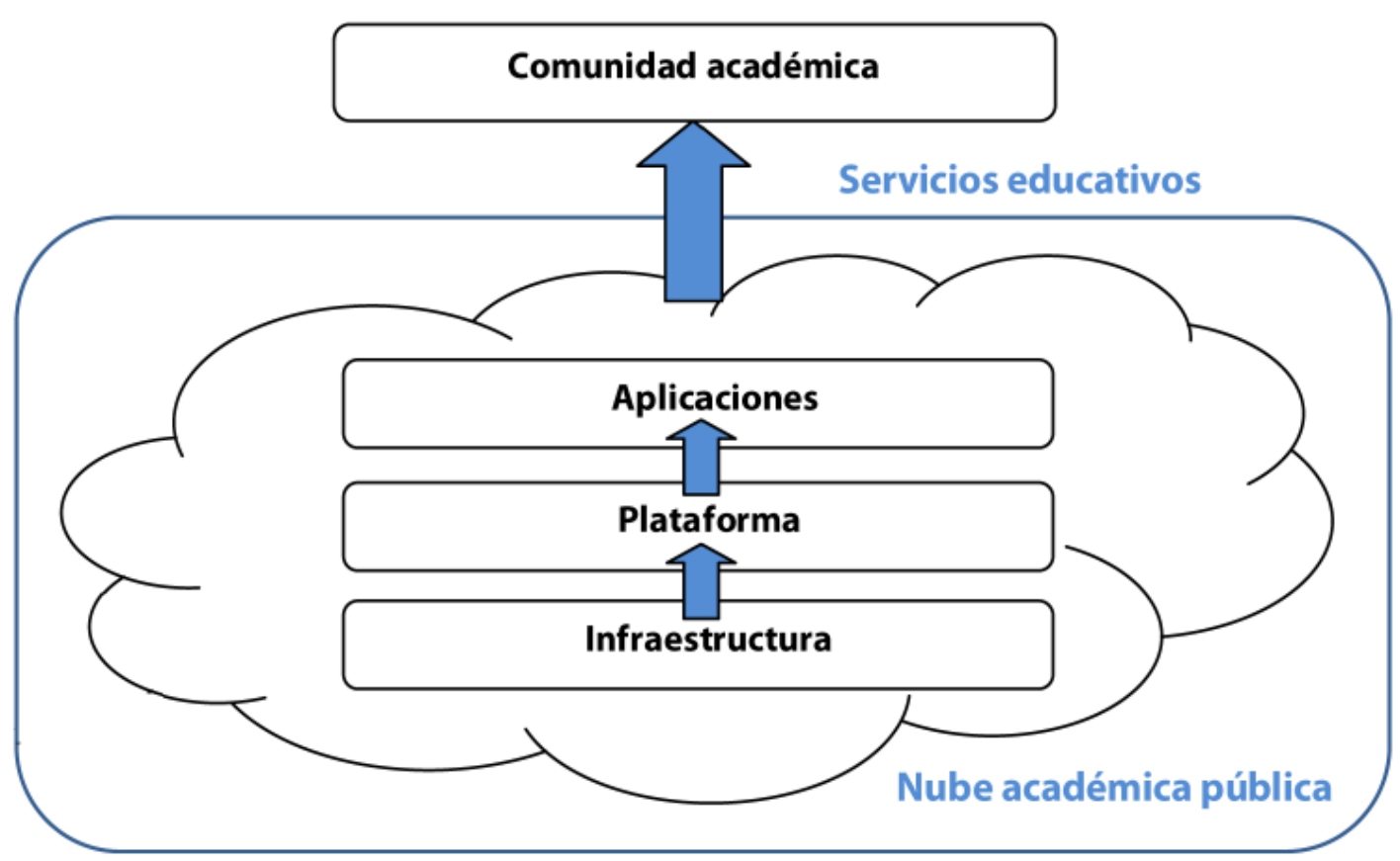

Figura 8. Recursos de la nube académica

Elaboración propia.

\section{B) Comunidad académica}

La comunidad académica está constituida por los estudiantes, docentes e investigadores de la institución universitaria que serán los usuarios de los recursos de información proporcionados por la Nube Académica en la forma de servicios, siguiendo los diversos modelos de Cloud Computing.

C) Procesos de las TI

Los procesos de las TI serán los encargados de administrar los recursos de la Nube Académica y nos ayudarán a determinar las mejores prácticas a ser utilizadas en su implantación y provisión de los servicios educativos. 
Tomando en consideración las características de las nubes públicas, así como las de los diferentes modelos asociados a él, y utilizando el marco de referencia del COBIT, se han identificado y priorizado siete procesos de las TI que nos ayudaran a definir el mapa de ruta para la implantación de estos servicios en el ámbito académico universitario. Estos procesos han sido seleccionados en base a la determinación de las metas de las TI relevantes de la institución educativa, conforme se muestra en la figura 9.

\section{Metas y procesos de las TI relevantes para la implantación de servicios de nube pública en una institución de ámbito académico universitario}

\begin{tabular}{|l|c|l|}
\hline $\begin{array}{l}\text { Metas de TI de la institución } \\
\text { para implantar servicios de } \\
\text { nube pública }\end{array}$ & $\begin{array}{c}\text { Proc. TI } \\
\text { Relevantes }\end{array}$ & Descripción del proceso \\
\hline $\begin{array}{l}\text { Determinar la dirección tecnológi- } \\
\text { ca para dar soporte a la institución } \\
\text { educativa, estableciendo expecta- }\end{array}$ & PO3 & Determinar la dirección tecnológica \\
\cline { 2 - 3 } $\begin{array}{l}\text { tivas realistas acerca de lo que la } \\
\text { tecnología puede ofrecer en tér- } \\
\text { minos de productos y servicios }\end{array}$ & PO9 & Administrar y evaluar riesgos \\
\hline $\begin{array}{l}\text { Identificar la solución de nube que } \\
\text { está alineada a los objetivos de la } \\
\text { institución }\end{array}$ & Al1 & Identificar soluciones automatizadas \\
\hline & Al4 & Facilitar la operación y uso \\
\cline { 2 - 3 } $\begin{array}{l}\text { Garantizar el uso adecuado de los } \\
\text { servicios educativos soportados } \\
\text { por tecnologías de nube pública } \\
\text { en la institución }\end{array}$ & DS1 & $\begin{array}{l}\text { Definir y administrar los niveles de } \\
\text { servicio }\end{array}$ \\
\cline { 2 - 4 } & ME2 & Administrar los servicios de terceros \\
\cline { 2 - 3 } & Monitorizar y evaluar el control interno \\
\hline
\end{tabular}

Figura 9. Metas y proceso de las TI

Elaboración propia. 
En la figura 10 se presenta el modelo de procesos de las TI definido:

\section{Planear y organizar}

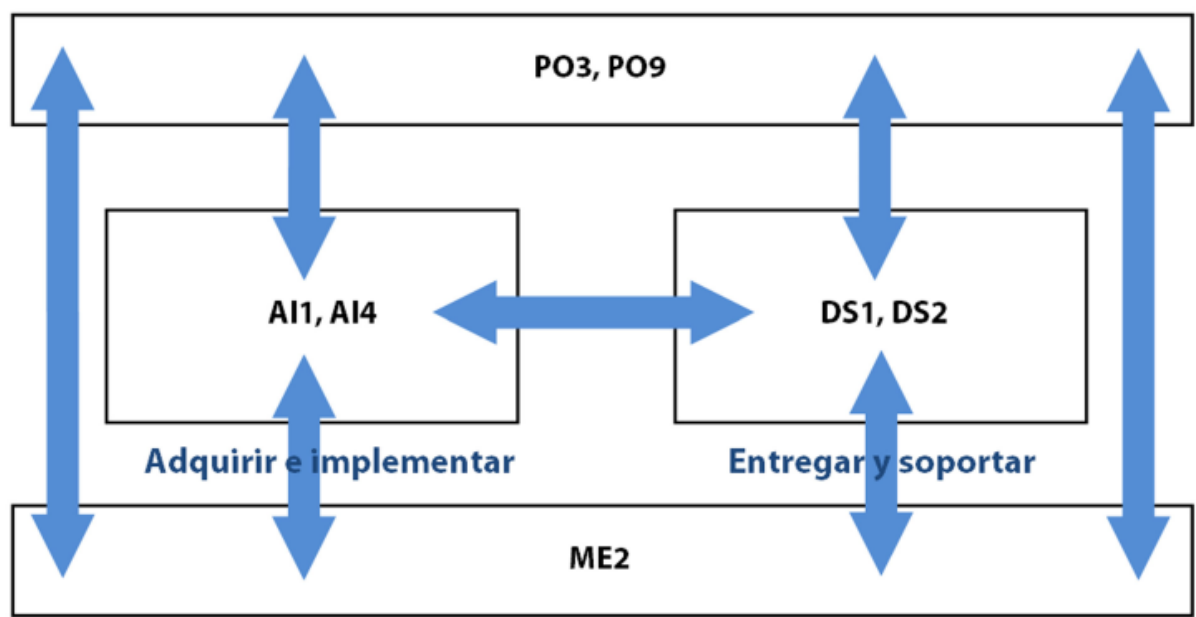

Monitorear y evaluar

Figura 10. Modelo de procesos de las TI para la nube pública

Fuente: Adaptado de ITGI. COBIT 4.1 (2007: 16).

Se muestra también el marco de trabajo definido para analizar el proceso de implantación de la Nube Académica pública en el ámbito universitario. 


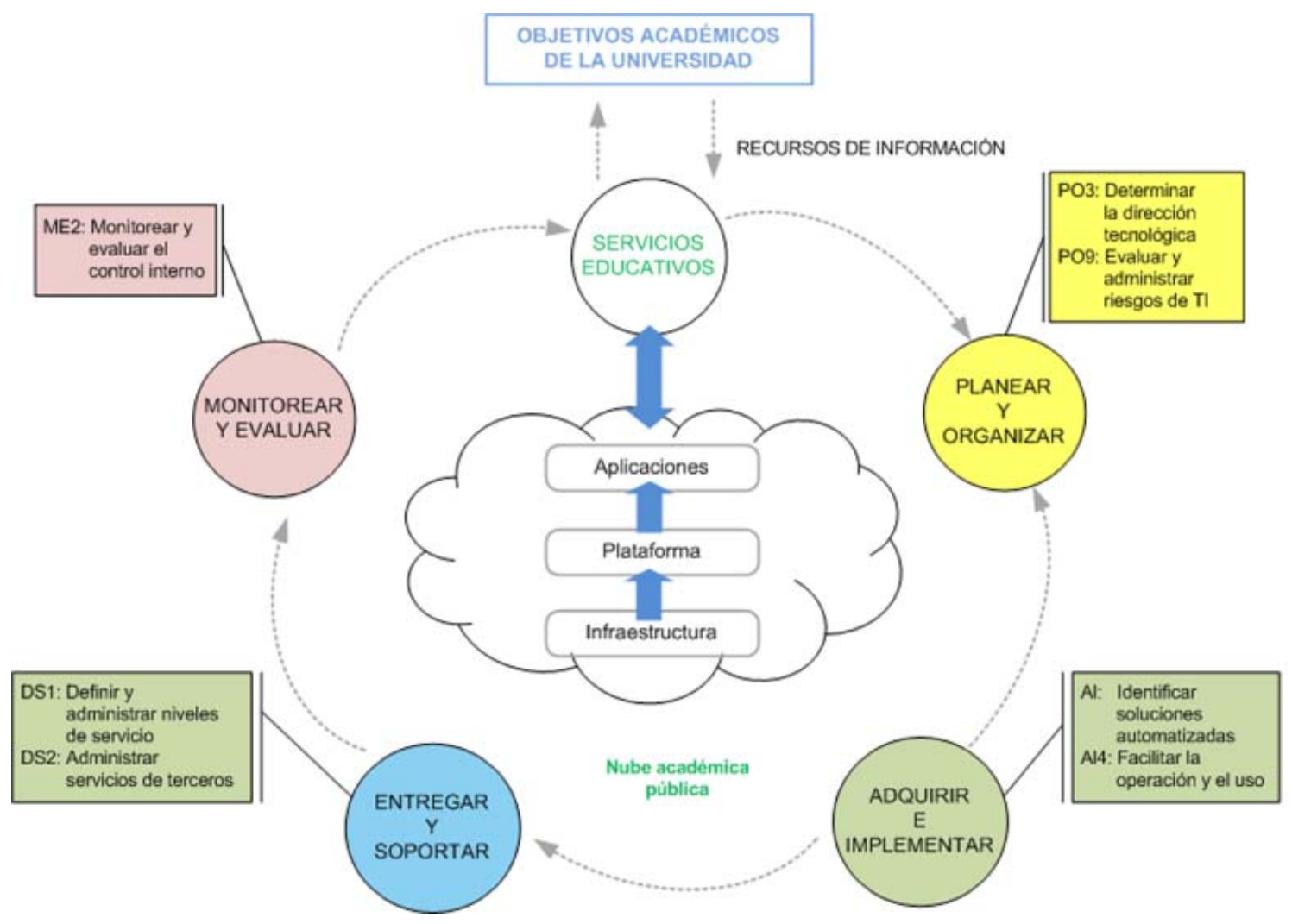

Figura 11. Marco de trabajo de la nube pública

Fuente: Adaptado de ITGI. COBIT 4.1 (2007: 26).

\section{Mapa de ruta para la implantación de la Nube Académica pública}

El mapa de ruta nos indica los proyectos de mejora (tareas, procedimientos, normas, etcétera) que deben ser implantados durante la etapa de iniciación con el fin de que los procesos de las TI seleccionados tengan un nivel de madurez adecuado.

Al lograrse este nivel de madurez en los procesos de las TI seleccionados se garantiza una gestión adecuada de los servicios educativos soportados por la nube.

Se logra el objetivo mediante la descripción de los procesos de las TI seleccionados y de los modelos de madurez determinados. Luego se seleccionan y describen los subprocesos más relevantes a ser considerados. Estas tareas se 
efectúan utilizando el documento COBIT 4.1. Information Technology Governance Institute (ITGI), para los siete procesos seleccionados.

Para cada uno de estos subprocesos se seleccionan los proyectos relevantes necesarios para su implantación con el nivel de madurez determinado. Se muestran también los beneficios para la institución académica universitaria y los riesgos que se evitarán como producto de implantar los proyectos seleccionados. Estas tareas se efectúan utilizando el documento COBIT. Control Practices. ITGI 2007, para los procesos y subprocesos seleccionados.

A continuación se muestran los cuadros resúmenes de los proyectos de mejora y del análisis de riesgo-valor correspondiente para los procesos el dominio de Planeary Organizar (PO). La implantación de estos proyectos de mejora servirá para que los procesos tengan un nivel de madurez adecuado para gestionar los recursos de TI de la nube. Se muestra también el valor que obtendrán las universidades, así como los riegos que evitarán, como consecuencia de la implantación de los mencionados proyectos.

De manera similar se efectuó el trabajo para los otros tres dominios del COBIT. Los resultados pueden encontrarse en el informe final de la investigación "Servicios educativos utilizando tecnologías de Cloud Computing", del Instituto de Investigación Científica de la Universidad de Lima (IDIC).

\section{PROYECTOS DE MEJORA DEL DOMINIO PLANEAR Y ORGANIZAR}

\begin{tabular}{|l|l|}
\hline Determinar la dirección tecnológica (PO3) & $\begin{array}{l}\text { Evaluar y administrar los riesgos de las } \\
\text { TI (PO9) }\end{array}$ \\
\hline $\begin{array}{l}\text { Desarrollar un análisis FODA para todos los ac- } \\
\text { tivos de las TI de forma regular }\end{array}$ & $\begin{array}{l}\text { Obtener acuerdo y aprobación de los } \\
\text { eventos claves y de sus impactos por parte } \\
\text { de los involucrados (stakeholders). }\end{array}$ \\
\hline $\begin{array}{l}\text { Hacer un seguimiento del mercado de las TI y } \\
\text { de las tecnologías emergentes relevantes }\end{array}$ & $\begin{array}{l}\text { Identificar los eventos potenciales que } \\
\text { pueden afectar negativamente a las me- } \\
\text { tas de la empresa o a sus operaciones, } \\
\text { considerando auditorías, inspecciones e } \\
\text { incidentes identificados, usando listas de } \\
\text { verificación, reuniones de trabajo (work- } \\
\text { shops), análisis de flujo de procesos y otras } \\
\text { técnicas y herramientas. }\end{array}$ \\
\hline $\begin{array}{l}\text { Identificar los últimos desarrollos en las TI que } \\
\text { pueden tener un impacto en el éxito del ne- } \\
\text { gocio }\end{array}$ & $\begin{array}{l}\text { Identificar impactos potenciales negativos } \\
\text { que sean relevantes y significativos para la } \\
\text { empresa, para cada uno de los eventos se- } \\
\text { leccionados. }\end{array}$ \\
\hline
\end{tabular}




\begin{tabular}{|c|c|}
\hline $\begin{array}{l}\text { Establecer el apetito de riesgo tecnológico } \\
\text { apropiado }\end{array}$ & $\begin{array}{l}\text { Determinar los umbrales de los riesgos } \\
\text { identificados de manera cualitativa (ejem- } \\
\text { plo: muy probable, probable, improbable) } \\
\text { o cuantitativa usando análisis estadístico y } \\
\text { probabilidades, basados en fuentes de in- } \\
\text { formación razonables que deben ser apro- } \\
\text { piadamente validadas. }\end{array}$ \\
\hline $\begin{array}{l}\text { Crear un plan de infraestructura tecnológica } \\
\text { basado en los planes estratégicos y tácticos } \\
\text { de } \mathrm{TI} \text { y la dirección tecnológica que inclu- } \\
\text { yan aspectos como tecnologías integradas } \\
\text { consistentes, arquitectura de los sistemas de } \\
\text { negocios, aspectos de contingencia de la in- } \\
\text { fraestructura y dirección para la adquisición } \\
\text { de activos de las TI. }\end{array}$ & $\begin{array}{l}\text { Determinar el impacto material en el ne- } \\
\text { gocio de los riesgos identificados de forma } \\
\text { cualitativa (ejemplo: catastrófico, crítico, } \\
\text { marginal) o cuantitativamente (ejemplo: } \\
\text { impacto en las utilidades o el valor de los } \\
\text { accionistas). }\end{array}$ \\
\hline \multirow[t]{3}{*}{$\begin{array}{l}\text { Efectuar una evaluación de los sistemas de } \\
\text { información actuales y proyectados y definir } \\
\text { una estrategia de migración o mapa de ruta } \\
\text { para lograr el estado futuro. }\end{array}$} & $\begin{array}{l}\text { Evaluar los riesgos inherentes al evento } \\
\text { considerando los controles que se utiliza- } \\
\text { rán para identificar el riesgo residual. }\end{array}$ \\
\hline & $\begin{array}{l}\text { Considerar el resultado de la evaluación } \\
\text { de riesgos y determinar una estrategia } \\
\text { para su mitigación, teniendo en cuenta el } \\
\text { significado del riesgo y el probable costo- } \\
\text { beneficio de las diversas opciones (evitar, } \\
\text { reducir, compartir y aceptar), que esté } \\
\text { alineado con los objetivos estratégicos } \\
\text { y de acuerdo con la cultura aceptada de } \\
\text { administración y tolerancia de riesgos de } \\
\text { la empresa. }\end{array}$ \\
\hline & $\begin{array}{l}\text { Desarrollar un plan de acción para imple- } \\
\text { mentar la respuesta a riesgos acordada. }\end{array}$ \\
\hline
\end{tabular}

Cuadro 1. Proyectos de mejora del dominio PO

Fuente: Adaptado de ITGI COBIT. Control Practices (2007). 


\section{ANÁLISIS DE VALOR DEL DOMINIO PLANEAR Y ORGANIZAR}

\begin{tabular}{|c|c|}
\hline $\begin{array}{l}\text { Determinar la dirección tecnológica } \\
\text { (PO3) }\end{array}$ & $\begin{array}{l}\text { Evaluar y administrar los riesgos } \\
\text { de las TI (PO9) }\end{array}$ \\
\hline $\begin{array}{l}\text { Mejorar el apalancamiento de la tecnolo- } \\
\text { gía para las oportunidades de negocio }\end{array}$ & $\begin{array}{l}\text { Enfoque consistente en la identificación } \\
\text { de eventos de riesgo }\end{array}$ \\
\hline $\begin{array}{l}\text { Mejorar la integración de la infraestructura } \\
\text { y las aplicaciones a través de los estándares } \\
\text { definidos por la dirección técnica }\end{array}$ & $\begin{array}{l}\text { Enfoque en los eventos de riesgo signifi- } \\
\text { cativos }\end{array}$ \\
\hline Mejor uso de los recursos y capacidades & $\begin{array}{l}\text { Mejora del planeamiento y uso de herra- } \\
\text { mientas y recursos de administración de } \\
\text { riesgos de las TI. }\end{array}$ \\
\hline $\begin{array}{l}\text { Reducción de los costos de las adquisicio- } \\
\text { nes técnicas a través de plataformas redu- } \\
\text { cidas y de una mejora en la administración } \\
\text { de las inversiones }\end{array}$ & $\begin{array}{l}\text { Credibilidad organizacional de la función } \\
\text { de evaluación de los riesgos de las TI. }\end{array}$ \\
\hline Mejora de la interoperabilidad & Administración efectiva de los riesgos \\
\hline $\begin{array}{l}\text { Mejora de las economías de escala para las } \\
\text { inversiones }\end{array}$ & $\begin{array}{l}\text { Enfoque consistente de mitigación de } \\
\text { riesgos }\end{array}$ \\
\hline $\begin{array}{l}\text { Plan tecnológico con buen balance en cos- } \\
\text { to, agilidad de los requerimientos y riesgo }\end{array}$ & Respuesta a riesgos efectiva en costos \\
\hline $\begin{array}{l}\text { Infraestructura tecnológica suficiente, es- } \\
\text { table y flexible para responder a los reque- } \\
\text { rimientos de información }\end{array}$ & \\
\hline
\end{tabular}

Cuadro 2. Análisis de valor para los proyectos del dominio PO

Fuente: Adaptado de ITGI. COBIT. Control Practices (2007). 
Servicios educativos mediante la utilización de tecnologías de Cloud Computing

\section{ANÁLISIS DE RIESGO DEL DOMINIO PLANEAR Y ORGANIZAR}

\begin{tabular}{|l|l|}
\hline $\begin{array}{l}\text { Determinar la dirección tecnológica } \\
\text { (PO3) }\end{array}$ & $\begin{array}{l}\text { Evaluar y administrar los riesgos } \\
\text { de las TI (PO9) }\end{array}$ \\
\hline $\begin{array}{l}\text { Adquisiciones tecnológicas inconsisten- } \\
\text { tes con los planes estratégicos }\end{array}$ & $\begin{array}{l}\text { Identificación de eventos de riesgo irre- } \\
\text { levantes y no enfoque en los riesgos ver- } \\
\text { daderamente relevantes }\end{array}$ \\
\hline $\begin{array}{l}\text { Infraestructura de Tl inapropiada para los } \\
\text { requerimientos de la organización }\end{array}$ & $\begin{array}{l}\text { Considerar importantes los riesgos irre- } \\
\text { levantes }\end{array}$ \\
\hline $\begin{array}{l}\text { Desviaciones de la dirección tecnológica } \\
\text { aprobada }\end{array}$ & $\begin{array}{l}\text { Riesgos vistos como eventos simples en } \\
\text { vez de verlos en su contexto global }\end{array}$ \\
\hline $\begin{array}{l}\text { Incremento de los costos debido a la falta } \\
\text { de coordinación y a un plan de adquisi- } \\
\text { ciones no estructurado }\end{array}$ & Riesgos significativos no considerados \\
\hline $\begin{array}{l}\text { Implantación de sistemas inconsistentes } \\
\text { Desviaciones de la dirección tecnológica } \\
\text { aprobada }\end{array}$ & Pérdida de activos de TI \\
\hline $\begin{array}{l}\text { Incremento de costos debido a planes de } \\
\text { adquisición no estructurados }\end{array}$ & $\begin{array}{l}\text { Riesgos de negocio residuales no iden- } \\
\text { tificados no efectiva }\end{array}$ \\
\hline
\end{tabular}

Cuadro 3. Análisis de riesgo para los proyectos del dominio PO

Fuente: Adaptado de ITGI. COBIT. Control Practices (2007) 


\section{Conclusiones y recomendaciones}

\subsection{Conclusiones}

1. La conclusión principal del presente estudio es que las tecnologías del Cloud Computing representan una oportunidad para cambiar el modelo de gestión y operación de las TI de las instituciones académicas universitarias, haciéndolas más eficientes y alineadas a sus objetivos estratégicos.

2. Las tecnologías del Cloud Computing están lo suficientemente maduras para ser implantadas en el ámbito universitario. Sin embargo, esta implantación debe ser gradual, llevarse a cabo con mucha cautela y en un proceso de aprendizaje continuo.

3. Las pruebas de concepto efectuadas nos permiten concluir que los servicios de Cloud Computing de Google Apps y Microsoft Live@edu son los más adecuados para su implantación en la etapa piloto propuesta.

\subsection{Recomendaciones}

1. Incluir las tecnologías de Cloud Computing como un elemento estratégico en la adopción de los futuros servicios en el ámbito académico universitario.

2. Las instituciones académicas universitarias deben iniciar al más breve plazo la implantación de la etapa piloto propuesta, esto les permitirá conocer y utilizar servicios educativos bajo los modelos de nube pública y nube privada.

3. Iniciar proyectos de mejora de los procesos de de las TI, relacionados con la provisión de servicios educativos en el ámbito académico universitario, utilizando el enfoque del Cloud Computing. Esta tarea puede ser acometida tomando como base los modelos de procesos de las TI y los proyectos de mejora planteados en esta investigación.

4. Analizar la viabilidad de implantar el enfoque de gobierno de las TI en el ámbito universitario, situación que garantizará el alineamiento de estas y los objetivos estratégicos de las universidades, la entrega de valor, la gestión del riesgo, el uso óptimo de los recursos y la medición de resultados. 


\section{BIBLIOGRAFÍA}

Badger, Lee y Tim Grance. "Standards acceleration to jumpstart adoption of Cloud Computing". http://csrc.nist.gov/groups/SNS/cloud-computing/

Educase/Nacubo (2010). "Shaping the higher education cloud". Workshop on Cloud Computing and above campus service. http://www.educause.edu/ Resources/ShapingtheHigherEducationCloud/205427.

Gartner (2010). "Gartner's enterprise architecture process and framework help meet $21^{\text {st }}$ century challenges". http://www.gartner.com/DisplayDocument?doc_ $\mathrm{cd}=133132$

Hofmann, Paul y Dan Woods. "Cloud Computing: The limits of public clouds for business applications". http://proquest.umi.com/pqdweb?did=2175071841 \&sid $=10 \&$ Fmt $=6 \&$ clientld $=46601 \& R Q T=309 \&$ VName $=P Q D$

Holschuh, Douglas R. y David C. Caverly. "Techtalk: Cloud Computing and developmental education". http://proquest.umi.com/pqdweb?did=206378 $8051 \&$ sid $=15 \& \mathrm{Fmt}=6 \&$ lientld $=46601 \& \mathrm{RQT}=309 \& \mathrm{VName}=\mathrm{PQD}$

Hurwitz, Judith; Bloor, Robin; Kaufman, Marcia y Fern Halper (2010). Cloud Computing for dummies. Indianapolis, Indiana: Wiley Publishing Inc.

IEEE (2009). "An E-Learning ecosystem based on Cloud Computing infrastructure". Ninth IEEE International Conference on Advanced Learning Technologies. http://ieeexplore.ieee.org/xpl/freeabs_all.jsp?arnumber=5194182

ITGI (2007). Information Technology Governance Institute. COBIT. Control Practices. Guidance to achieve Control Objectives for successful IT Governance. $2^{\text {nd }}$ Edition.

ITGI. Information Technology Governance Institute. COBIT 4.1. http://www.isaca. org/Knowledge-Center/cobit/Pages/Downloads.aspx.

itSMF (2006). The IT Service Management Forum "Frameworks for IT Management". Van Haren Publishing.

Katzan, Harry. "The educational value of Cloud Computing". http://proquest.umi. $\mathrm{com} /$ pqdweb?did $=2097983941 \&$ sid $=15 \& \mathrm{Fmt}=6 \& \mathrm{client} \mathrm{d}=46601 \& \mathrm{RQT}=30$ $9 \& \mathrm{VName}=\mathrm{PQD}$

Leaf, Dawn (2010). "Overview: NIST Cloud Computing Efforts". http://csrc.nist.gov/ groups/SNS/cloud-computing/

Minoli, Daniel (2008). “Enterprise architecture A to Z: Frameworks, business process modeling, SOA, and infraestructure technology". Boca Ratón: CRC Press. 
Pallis, George (2010). "Cloud Computing. The new frontier of internet computing". http://proquest.umi.com/pqdweb?did=2123126421\&sid=13\&Fmt=6\&clien $\mathrm{tl} d=46601 \& \mathrm{RQT}=309 \& \mathrm{VName}=\mathrm{PQD}$

Tseronis, Pete; Lewin, Katie; Garbars, Kurt y Peter Mell."Federal risk and authorization management program". http://csrc.nist.gov/groups/SNS/cloudcomputing/.

Zhang, Liang-Jie; Zhang, Jia; Fiaidhi, Jinan y J. Morris Chang. "Hot topics in Cloud Computing". http://proquest.umi.com/pqdweb?did=2149253181\&sid=5\&F $\mathrm{mt}=6 \&$ clientld $=46601 \& \mathrm{RQT}=309 \& \mathrm{VName}=\mathrm{PQD}$ 